\title{
MộT PHƯƠNG PHÁP TẠO MẶT CHO ĐỐI TƯợNG 3D
}

\author{
LÊ QUANG HÙNG(1), TRÀ̀ THÙY DƯƠNG ${ }^{(2)}$ \\ ${ }^{(1)}$ Công ty Cổ phần Công nghệ Tài nguyên Môi trường và Vật liệu \\ ${ }^{(2)}$ Trường Đại học Mỏ - Địa chất
}

\section{Tóm tắt:}

Trong mặt phẳng, khái niệm vùng sủ dụng để mô tả một đa giác khép kín được tạo nên tù dũ liệu ban đầu gồm các phần tủ co sở là điểm và đoạn. Để tìm, quản lý nó nguời ta sủ dụng bài toán tạo vùng. Bề mặt mồt đối tượng $3 D$ bao gồm một tập hợp các mặt mà mỗi mặt được mô hình hóa bằng một đa giác phẳng khép kín. Các mặt này cũng tương tụ như các vùng trong mặt phẳng nhung được biểu diễn trong không gian 3D. Bài toán tạo vùng trong mặt phẳng được nghiên cứu giải quyết và được kiểm chứng bằng lý thuyết cũng nhu thực tiển áp dụng để khoanh, tính diện tích thửa đất trong bản đồ địa chính. Trên co sở bài toán này, bài báo nghiên cúu và đề xuất một phuơng pháp tạo mặt để mô tả bề mặt đối tuợng 3D. Bài toán này áp dụng nguyên tắc một cạnh chỉ có hai mặt liền kề; tùt một cạnh biên tìm được cạnh biên tiếp theo. Bài toán sẽ phân loại các cạnh của khối $3 D$, sau đó chiếu chúng về mặt phẳng. Việc phân loại nhằm đonn giản bài toán không gian bằng cách biến đổi nó thành bài toán giải quyết trên mặt phẳng. Tuy nhiên, khi chiếu các cạnh về mặt phẳng, việc sắp xếp các cạnh chiếu theo thư tụ nhu trong không gian là vấn đề không đơn giản. Nghiên cứu này chỉ ra có thể sắp xếp được nhu trên khi các mặt thỏa mãn điều kiện nhất định. Bài toán tạo vùng trên mặt phẳng sẽ tạo được các mặt tương ứng bề mặt của đối tượng $3 D$ trong không gian.

\section{1. Đặt vấn đề}

Hiện nay, công nghệ máy tính đã đạt được những bước phát triển vượt bậc với những tính năng như tốc độ xử lý nhanh, dung lượng bộ nhớ lớn... mạnh hơn rất nhiều so với trước đây. Xu hướng mô hình hóa các đối tượng $3 \mathrm{D}$ trong những năm gần đây được lựa chọn để biểu diễn mô hình dữ liệu không gian đang dần trở nên phổ biến hơn. Trong lĩnh vực quản lý đô thị nói riêng thì đối tượng thửa đất là một đối tượng quản lý hết sức quan trọng nhưng cũng rất phức tạp. Hiện tại người ta mới chỉ quản lý được ranh giới của thửa đất còn các đối tượng khác như các công trình trên đất vẫn sử dụng các phương pháp truyền thống dạng 2D. Các đối tượng khi được mô hình hóa và quản lý trong không gian $3 \mathrm{D}$ sẽ giúp cho các nhà quản lý xử lý "thông minh" hơn khi giải quyết các bài toán của mình.Các công trình trên đất thường là các đối tượng kiến trúc hình khối (tòa nhà cao tầng) cần thiết phải biểu diễn theo mô hình không gian 3D. Việc nghiên cứu tạo mặt cho khối 3D cũng tương tự như việc khoanh các mặt của tòa nhà nhằm mô hình hóa các khối kiến trúc phục vụ cho công tác quản lý đô thị trong lĩnh vực xây dựng. Mục tiêu hướng tới của bài toán là xây dựng liên kết giữa dữ liệu ban đầu là các điểm, cạnh (dữ liệu đo đạc) và bài toán khoanh khối đã được nghiên cứu trong công trình [8] phục vụ cho mô hình hóa các đối tượng trong quản lý đô thị và nghiên cứu xây dựng cơ sở dữ liệu đất đai.

Khi quan sát khối 3D được mô hình hóa cho thấy bề mặt đối tượng được tạo bởi các mặt và các mặt được tạo bởi các điểm và đoạn (cạnh). Trong mặt phẳng khi xác định được điểm và cạnh sẽ thu được các vùng còn trong không gian thu được các mặt của đối tượng.

Giả sử, có một đối tượng 3D bao gồm các mặt liên thông với nhau. Mỗi một mặt có thể coi là một tập hợp bởi chuỗi cạnh biên hay điểm biên của một đa giác phẳng khép kín. Việc mô hình hóa mặt phẳng trong không gian tương tự như xác định một vùng trong mặt phẳng. 
Dữ liệu đầu vào gồm có: điểm, cạnh trên bề mặt của đối tượng 3D.

Bài báo này giải quyết bài toán đi tìm các mặt hay các đường đa giác trên bề mặt của đối tượng 3D đó.

Hướng giải quyết bài toán tìm mặt cho đối tượng 3D trong không gian lấy bài toán tạo vùng trong mặt phẳng làm cơ sở.

Bài toán tạo vùng đã được trình bày trong các công trình [2], [3].

Nội dung nghiên cứu nhằm đề xuất một phương pháp giải bài toán mặt phục vụ cho giải quyết các bài toán không gian có độ phức tạp cao trên mô hình $3 \mathrm{D}$ có nhiều ứng dụng trong thực tiễn như bài toán tính thể tich, tính khối lượng đào đắp ...

\section{Giải quyết vấn đề: Bài toán tìm mặt cho đối tượng 3D}

\subsection{Nguyên tắc giải quyết bài bài toán}

- Mỗi cạnh chỉ có 2 bề mặt liền kề hay nói cách khác mỗi nửa cạnh chỉ có duy nhất một mặt kề với nó.

- Các bề mặt xét trong bài toán thỏa mãn điều kiện hàm đơn ánh.

Hàm đơn ánh: Với mọi $\mathrm{x} 1$ và $\mathrm{x} 2$ thuộc $\mathrm{X}$ và nếu $\mathrm{x} 1 \neq \mathrm{x} 2$ thì $\mathrm{f}(\mathrm{x} 1) \neq \mathrm{f}(\mathrm{x} 2)$.

Có nghĩa là bề mặt 3D (hay bề mặt địa hình) không có đường đứt gãy (hàm ếch).

- Trong mặt phẳng nếu biết được 1 cạnh có thể tìm được cạnh tiếp theo. Trong không gian cũng tìm cạnh theo nguyên tắc này.

Áp dụng nguyên tắc này, để sắp xếp các cạnh trong không gian, đem chiếu toàn bộ các cạnh xuống mặt phẳng. Nếu thỏa mãn hàm Đơn ánh thì thứ tự sắp xếp các cạnh chiếu trên mặt phẳng sẽ tuân theo thứ tự sắp xếp của các mặt trong không gian.

\subsection{Các bước thục hiện}

Giả sử có khối 3D như dưới hình 1 dưới đây

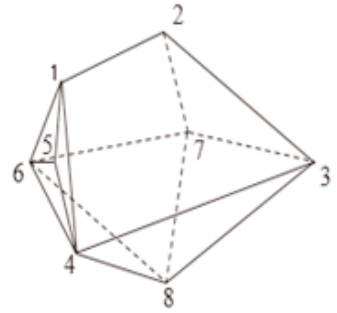

Hình 1: Đối tượng 3D

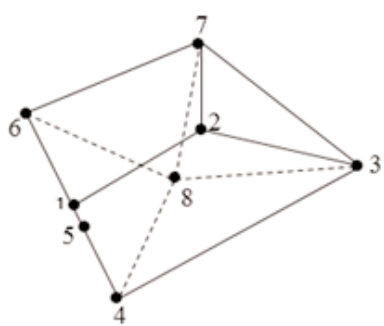

Hình 2: Hình chiếu trên mặt phẳng

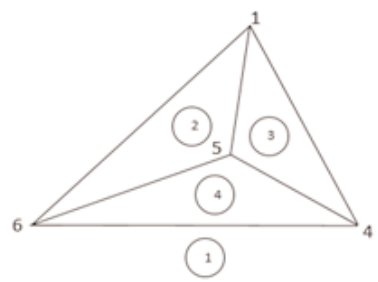

Hình 3: Các cạh “Đứng”

\section{Bước 1: Tìm bao lồi}

Để tìm bao lồi, thực hiện chiếu tất cả các điểm, cạnh của khối $3 \mathrm{D}$ xuống mặt phẳng. Trên mặt phẳng thu được một bao lồi chứa hình chiếu tất cả các điểm, các cạnh. Các điểm, cạnh này có thể nằm phía "Trên" hoặc phía "Dưới” hoặc có thể nằm ngay trên cạnh của bao lồi.

Trên hình 2 bao lồi thu được là đa giác khép kín gồm tập hợp các điểm: 1, 6, 7, 3, 4, 5

Quan sát một khối 3D (Hình 1), có thể nhận thấy rằng các điểm, đoạn của các mặt tạo nên khối 3D có thể phân chia thành 3 loại như sau:

- Các điểm, cạnh nằm ở phía “Trên” khối.

- Các điểm, cạnh nằm ở phía "Dưới” khối.

- Các điểm, cạnh nằm trên mặt "Đứng” khối.

Về cạnh, cũng phân thành các loại sau: 
- Cạnh phía trên bao lồi. Ký hiệu cạnh là "Trên".

- Cạnh trên bao lồi. Ký hiệu là cạnh "Lồi”.

- Cạnh thuộc mặt “Đứng”, ký hiệu là cạnh "Đứng"

- Cạnh phía dưới bao lồi. Ký hiệu là cạnh "Dưới"

Việc phân loại này nhằm mục đích khi xử lý với các điểm, cạnh nằm ở phía "Trên" khối sẽ thu được các mặt “Trên”, với các điểm, cạnh nằm ở phía "Dưới” khối sẽ thu được các mặt "Dưới" và với các điểm, cạnh nằm ở mặt "Đứng” khối thu được các mặt "Đứng".

\section{Bước 2: Chọn Zmax.}

Chọn 1 điểm bất kì có giá trị $Z=Z m a x$. Điểm có Zmax chắc chắn sẽ là điểm nằm phía trên bao lồi và thuộc mặt trên, thu được cạnh "Trên Trên".

Bước 3: Tìm các cạnh nằm phía trên, bao lồi, dưới bao lồ và sắp xếp theo danh sách nưa cạn trên, nứa cạnh dưới và nưa cạnh đứng

Áp dụng nguyên tắc biết được 1 cạnh, tìm được cạnh tiếp theo. Dựa vào Zmax tìm được các cạnh "Trên - Trên" và tiếp tục tìm; nếu cạnh tiếp theo là cạnh "Trên - Lồi" thì dừng lại và xếp chúng vào nhóm nửa cạnh trên, tiếp tục tìm kiếm nếu cạnh tìm được là cạnh "Lồi - Lồi”, xếp cạnh đó vào nhóm nửa cạnh đứng; nếu cạnh tìm được là cạnh "Dưới - Lồi” sắp xếp cạnh đó vào nhóm nửa cạnh dưới.

Tiến hành tương tự như vậy sẽ thu được một danh sách ban đầu gồm các nửa cạnh trên, các nửa cạnh đứng là cạnh của bao lồi và nhóm nửa các cạnh dưới.

\section{Bước 4: Tìm và sắp xếp các cạnh "Đứng”}

Các mặt đứng là các mặt có tính chất: Chứa một cạnh của bao lồi và hình chiếu của nó trên mặt phẳng là một đoạn thẳng. Để tìm các cạnh "Đứng" sử dụng hệ số góc $\frac{d x}{d z}$ của cạnh bao lồi.
Giả sử chọn cạnh của bao lồi 1-6 (Hình 1) làm gốc, tính được giá trị $\frac{d x(1,6)}{d z(1,6)}$; tiếp tục lấy các cạnh tiếp theo là cạnh có điểm đầu là điểm 1 điểm cuối giả sử là điểm thứ $\mathrm{i}(\mathrm{i}=1 \rightarrow \mathrm{n})$ và tính hệ số $\left(\frac{d x(1, i)}{d z(1, i)}\right)$. So sánh với hệ số $\frac{d x(1,6)}{d z(1,6)}$.

Nếu: $\frac{d x(1,6)}{d z(1,6)}=\frac{d x(1, i)}{d z(1, i)} \quad$ tức là điểm thứ i hay cạnh 1-i đó cùng thuộc mặt đứng chứa cạnh 1-6. Làm tương tự lần lượt cho tất cả các điểm còn lại, xác định được tất cả các điểm, cạnh nằm trên mặt phẳng “Đứng” chứa cạnh lồi 1-6. Cũng làm tương tự như vậy cho các cạnh còn lại trên bao lồi sẽ xác định được tất cả các điểm, cạnh nằm thuộc các mặt đứng.

Sau khi xác định được các cạnh thuộc các mặt "Đứng" tiến hành chiếu tất cả các cạnh này lên mặt ZOX hay ZOY thu được một danh sách các cạnh thuộc mặt "Đứng” (Hình 3). Sử dụng bài toán tạo Topo để khoanh vùng như trong mặt phẳng được một danh sách các vùng. Trong các vùng thu được, tồn tại một vùng biên và bài toán xử lý vùng biên đã được giải quyết trong công trình [8]. Theo đó, để gắn dấu cho kết quả tính diện tích của các hình thang con có thể dựa (xét) theo dấu của diện tích hình thang. Nếu diện tích hình thang con (là cạnh thuộc đường biên) tương ứng mang dấu dương thì cạnh bao lồi sẽ được sắp vào nửa cạnh trên, còn khi cạnh ứng với diện tích hình thang con mang dấu âm cạnh bao lồi đó sẽ sắp vào nửa cạnh dưới. Tuy nhiên, để thỏa mãn điều kiện khi khoanh vùng tạo mặt phẳng phải là hình đa giác khép kín nên cạnh thuộc bao lồi phải sắp cả hai nửa cạnh vào nửa cạnh trên và nửa cạnh dưới.

Sau khi tìm được các cạnh "Lồi” vào các nửa cạnh trên, nửa cạnh dưới và các nửa cạnh đứng, các cạnh thuộc tập hợp "Lồi" còn lại chưa được sắp xếp sẽ sắp chúng vào cả 3 tập hợp nửa cạnh trên, nửa cạnh dưới và nửa cạnh đứng.

Đến đây chúng ta đã hoàn thành việc phân chia các cạnh của đối tượng $3 \mathrm{D}$ vào 3 nhóm: các 
nửa trên cạnh thuộc các mặt "Trên", các nửa cạnh dưới thuộc các mặt "Dưới” và các nửa cạnh đứng thuộc các mặt "Đứng”. Với cách phân chia thành các nhóm cạnh này đã biến đổi từ bài toán không gian về bài toán cơ bản: Tạo vùng trong mặt phẳng.

Bước 5: Tiến hành tạo Topo từ nhóm các cạnh thuộc các tập hợp nửa cạnh trên sẽ thu được các mặt "Trên"; Topo từ nhóm các cạnh thuộc các tập hợp nửa cạnh dưới, thu được các mặt "Dưới”, Topo từ nhóm các cạnh thuộc các tập hợp nửa cạnh đứng thu được các mặt "Đứng" của một đối tượng 3D.

Kết thúc thuật toán.

\section{Thực nghiệm}

\subsection{Diễn giải thục nghiệm}

Xét khối 3D như hình 1

Dữ liệu ban đầu: Danh sách điểm có tọa độ: (Xem bảng 1)

- Danh sách các cạnh:

\begin{tabular}{|c|c|c|}
\hline \multicolumn{3}{|c|}{ Cạnh } \\
\hline ST T & ID1 & ID2 \\
\hline 1 & 1 & 2 \\
2 & 1 & 4 \\
3 & 1 & 6 \\
4 & 1 & 5 \\
5 & 2 & 3 \\
6 & 2 & 7 \\
7 & 3 & 7 \\
8 & 3 & 4 \\
9 & 3 & 8 \\
10 & 4 & 5 \\
11 & 4 & 6 \\
12 & 4 & 8 \\
13 & 5 & 6 \\
14 & 6 & 7 \\
15 & 6 & 8 \\
16 & 7 & 8 \\
\hline
\end{tabular}

- Danh sách cạnh, nhân đôi (nửa cạnh):

\begin{tabular}{|c|c|c|}
\hline \multicolumn{3}{|c|}{ Nửa cạnh } \\
\hline Cạnh & ID1 & ID2 \\
\hline 1 & 1 & 2 \\
2 & 1 & 4 \\
3 & 1 & 6 \\
4 & 1 & 5 \\
5 & 2 & 3 \\
6 & 2 & 7 \\
7 & 3 & 7 \\
8 & 3 & 4 \\
9 & 3 & 8 \\
10 & 4 & 5 \\
11 & 4 & 6 \\
12 & 4 & 8 \\
13 & 5 & 6 \\
14 & 6 & 7 \\
15 & 6 & 8 \\
16 & 7 & 8 \\
\hline
\end{tabular}

\begin{tabular}{|c|c|c|}
\hline \multicolumn{3}{|c|}{ Nừa cạnh } \\
\hline Cạnh & ID1 & ID2 \\
\hline 17 & 2 & 1 \\
18 & 4 & 1 \\
19 & 6 & 1 \\
20 & 5 & 1 \\
21 & 3 & 2 \\
22 & 7 & 2 \\
23 & 7 & 3 \\
24 & 4 & 3 \\
25 & 8 & 3 \\
26 & 5 & 4 \\
27 & 6 & 4 \\
28 & 8 & 4 \\
29 & 6 & 5 \\
30 & 7 & 6 \\
31 & 8 & 6 \\
32 & 8 & 7 \\
\hline
\end{tabular}

\section{Bước 1: Tìm bao lồi}

Đem chiếu tất cả cả các cạnh của khối 3D xuống mặt XOY như hình 2 thu được bao lồi là một hình đa giác khép kín đi qua các đỉnh: 1,6 , $7,3,4,5$.

\section{Buớc 2: chon Zmax}

Nhìn vào bảng thống kê tọa độ theo danh sách điểm dễ dàng thấy điểm 2 có trị số $Z$ lớn nhất. Chọn điểm có Zmax là điểm 2.

Sau khi chọn được Zmax, dựa vào danh sách nửa cạnh và dùng màu để phân biệt, lập được Xâu kề của các cạnh như sau:

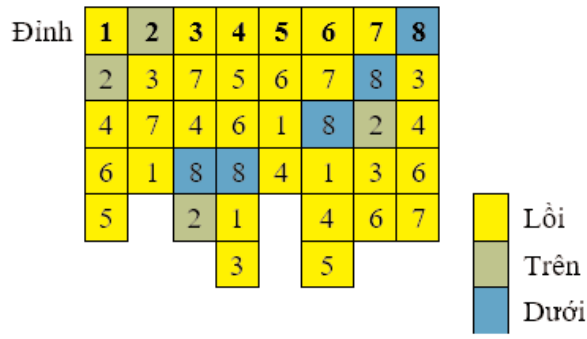

Bảng 1

\begin{tabular}{|c|c|c|c|c|c|c|c|c|}
\hline Điềm & 1 & 2 & 3 & 4 & 5 & 6 & 7 & 8 \\
\hline $\mathrm{X}(\mathrm{m})$ & 38.50 & 56.00 & 70.00 & 31.00 & 40.00 & 46.00 & 70.00 & 54.00 \\
\hline $\mathrm{Y}(\mathrm{m})$ & 29.00 & 24.00 & 00.00 & 15.00 & 32.00 & 43.00 & 30.00 & 17.00 \\
\hline $\mathrm{Z}(\mathrm{m})$ & 30.00 & 35.00 & 10.00 & 10.00 & 17.37 & 10.00 & 10.00 & 00.00 \\
\hline
\end{tabular}




\section{Bước 3:}

Dựa vào Xâu kề trên, sắp xếp theo nhóm nửa cạnh trên, nửa cạnh dưới, nửa cạnh đứng theo nguyên tắc:

- Cạnh "Trên-Trên" và Cạnh "Trên-Lồi” chắc chắn sẽ nằm phía trên bao lồi, sắp vào nhóm nửa cạnh trên,

- Cạnh "Lồi-Lồi” chắc chắn sẽ nằm thuộc mặt đứng chứa cạnh bao lồi, sắp vào nhóm nửa cạnh đứng,

- Cạnh "Dưới-Lồi” chắc chắn sẽ nằm phía dưới bao lồi, sắp vào nhóm nửa cạnh dưới.

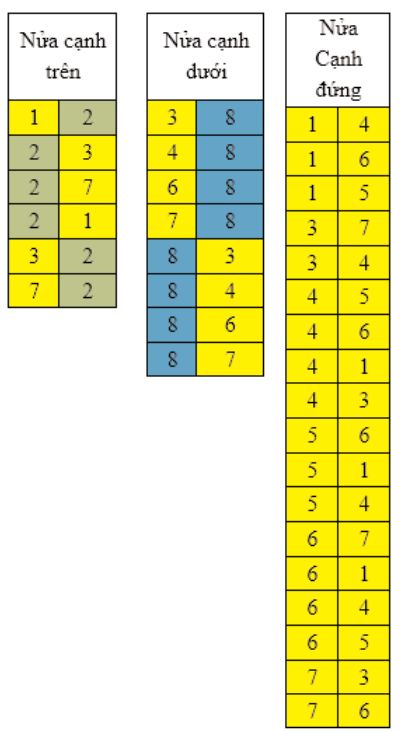

Bước 4: Tìm các cạnh "Đứng". Từ các nửa cạnh đứng, lập Xâu kề cạnh "Đứng"

\begin{tabular}{|l|l|l|l|l|l|}
\hline $\mathbf{l}$ & $\mathbf{3}$ & $\mathbf{4}$ & $\mathbf{6}$ & $\mathbf{7}$ & $\mathbf{5}$ \\
\hline 4 & 7 & 5 & 7 & 3 & 6 \\
\hline 6 & 4 & 6 & 1 & 6 & 1 \\
\hline 5 & & 1 & 4 & & 4 \\
\cline { 1 - 4 } & 3 & 5 & \multicolumn{3}{|c}{} \\
\cline { 3 - 4 } & &
\end{tabular}

Từ Xâu kề thu được các cạnh của mặt đứng:

Cạnh của mặt đứng
\begin{tabular}{|c|c|}
1 & 4 \\
1 & 6 \\
1 & 5 \\
4 & 5 \\
4 & 6 \\
4 & 1 \\
5 & 6 \\
5 & 1 \\
5 & 4 \\
6 & 1 \\
6 & 4 \\
6 & 5 \\
\hline
\end{tabular}$\quad$\begin{tabular}{|c|c|}
1 & 6 \\
1 & 5 \\
1 & 4 \\
4 & 1 \\
4 & 5 \\
4 & 6 \\
5 & 1 \\
5 & 6 \\
5 & 4 \\
6 & 1 \\
6 & 4 \\
6 & 5 \\
\hline
\end{tabular}

Chiếu các cạnh của mặt đứng lên mặt phẳng ZOX, dùng bài toán tạo vùng như trong mặt phẳng thu được các vùng như sau:
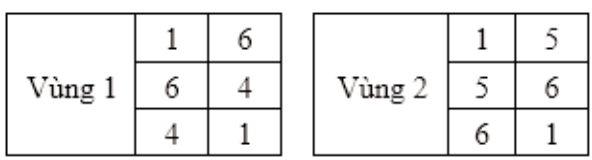

\begin{tabular}{|l|l|l|}
\hline \multirow{4}{*}{ Vùng 3} & 1 & 4 \\
\cline { 2 - 3 } & 4 & 5 \\
\cline { 2 - 3 } & 5 & 1 \\
\hline
\end{tabular}

\begin{tabular}{|l|l|l|}
\hline \multirow{2}{*}{ Vùng 4 } & 4 & 6 \\
\cline { 2 - 3 } & 6 & 5 \\
\cline { 2 - 3 } & 5 & 4 \\
\hline
\end{tabular}

Trong 4 vùng thu được có 1 vùng gọi là vùng biên (Vùng 1, hình 3 ). Vùng biên là vùng giống như một bao lồi trên mặt chiếu "Đứng" ZOX. Vùng biên có đặc điểm là vừa có các cạnh "Trên" vừa có cạnh "Dưới". Để phân loại và sắp xếp dựa vào cách xét dấu đã được nghiên cứu trong công trình [8].

Xét vùng biên:

\begin{tabular}{|c|c|c|c|c|c|c|c|c|}
\hline ID1 & ID2 & $\mathrm{x} 1$ & $\mathrm{z} 1$ & $\mathrm{x} 2$ & $\mathrm{z} 2$ & $\mathrm{x} 2-\mathrm{x} 1$ & $\mathrm{z} 1+\mathrm{z} 2$ & Diện tích \\
\hline 1 & 6 & 38.5 & 30.0 & 46.0 & 10.0 & 7.5 & 40.0 & 150 \\
6 & 4 & 46.0 & 10.0 & 31.0 & 10.0 & -15.0 & 20.0 & -150 \\
4 & 1 & 31.0 & 10.0 & 38.5 & 30.0 & 7.5 & 40.0 & 150 \\
\hline
\end{tabular}

Theo công trình [8], cạnh 1-6 và cạnh 4-1 mang dấu dương, sắpvào tập hợp nửa cạnh trên; cạnh 6-4 mang dấu âm, sắpvào tập hợp nửa cạnh dưới.

Do các cạnh 1-6, 4-1, 6-4 nằm trên bao lồi, để đủ điều kiện khép kín cho khoanh vùng phải sắp cả hai nửa cạnh $(1-6,6-1 ; 4-1,1-4)$ vào tập hợp nửa cạnh trên và sắp cả hai nửa cạnh (6-4 và 4 6) vào tập hợp nửa cạnh dưới.

Đến đây chúng ta đã hoàn thành phân loại và sắp xếp xong các cạnh của vùng (mặt) biên và các cạnh tạo thành vùng (mặt) trên mặt "Đứng".

Các cạnh đứng còn lại (cạnh nằm trên bao lồi) chưa được sử dụng sẽ vừa thuộc mặt "Trên" và vừa thuộc mặt "Dưới” và vừa thuộc mặt "Đứng" sẽ sắp đồng thời các nửa cạnh vào các tập hợp nửa cạnh trên, nửa cạnh dưới.

Sau sắp xếp sẽ thu được đầy đủ các nửa cạnh trên thuộc mặt "Trên", nửa cạnh dưới thuộc mặt "Dưới” và nửa cạnh đứng thuộc mặt "Đứng” và sắp lại các cạnh của nửa cạnh trên, nửa cạnh 
dưới như sau: (Xem bảng 2)

Bước 5: Áp dụng bài toán tạo vùng thu được các mặt - Vùng như sau: (Xem bảng 3)

Tóm lại: Khi tiến hành tạo mặt cho đối tượng 3D như hình 1, chúng ta thu được tập hợp 13 mặt tạo nên khối 3D. Trong đó:
-Mặt “Trên” khối gồm 4 mặt,

-Mặt "Đứng" khối gồm 5 mặt,

-Mặt "Đứng" khối gồm 5 mặt.

3.2. Thục nghiệm trên modul chương trình tính toán

Bước 1: Tìm Bao Lồi

Bảng 2

\begin{tabular}{|c|c|c|c|}
\hline \multicolumn{2}{|c|}{$\begin{array}{c}\text { Nừa cạnh } \\
\text { trên }\end{array}$} & \multicolumn{2}{|c|}{$\begin{array}{c}\text { Nửa Cạnh } \\
\text { dưới }\end{array}$} \\
\hline 1 & 2 & 3 & 8 \\
\hline 2 & 3 & 4 & 8 \\
\hline 2 & 7 & 6 & 8 \\
\hline 2 & 1 & 7 & 8 \\
\hline 3 & 2 & 8 & 3 \\
\hline 7 & 2 & 8 & 4 \\
\hline 1 & 6 & 8 & 6 \\
\hline 4 & 1 & 8 & 7 \\
\hline 6 & 1 & 6 & 4 \\
\hline 1 & 4 & 4 & 6 \\
\hline 3 & 7 & 3 & 7 \\
\hline 7 & 3 & 7 & 3 \\
\hline 3 & 4 & 3 & 4 \\
\hline 4 & 3 & 4 & 3 \\
\hline 6 & 7 & 6 & 7 \\
\hline 7 & 6 & 7 & 6 \\
\hline
\end{tabular}

\begin{tabular}{|c|c|}
\hline \multicolumn{2}{|c|}{$\begin{array}{c}\text { Nửa Cạnh } \\
\text { đừng }\end{array}$} \\
\hline 1 & 4 \\
\hline 1 & 6 \\
\hline 1 & 5 \\
\hline 3 & 7 \\
\hline 3 & 4 \\
\hline 4 & 5 \\
\hline 4 & 6 \\
\hline 4 & 1 \\
\hline 4 & 3 \\
\hline 5 & 6 \\
\hline 5 & 1 \\
\hline 5 & 4 \\
\hline 6 & 7 \\
\hline 6 & 1 \\
\hline 6 & 4 \\
\hline 6 & 5 \\
\hline 7 & 3 \\
\hline 7 & 6 \\
\hline & \\
\hline
\end{tabular}

\begin{tabular}{|c|c|}
\hline \multicolumn{2}{|c|}{$\begin{array}{c}\text { Nừa cạh trên } \\
\text { sắp lại }\end{array}$} \\
\hline 1 & 2 \\
1 & 6 \\
1 & 4 \\
2 & 7 \\
2 & 1 \\
2 & 3 \\
3 & 7 \\
3 & 2 \\
3 & 4 \\
4 & 1 \\
4 & 3 \\
6 & 7 \\
6 & 1 \\
7 & 6 \\
7 & 2 \\
7 & 3 \\
\hline
\end{tabular}

\begin{tabular}{|c|c|}
\hline \multicolumn{2}{|c|}{$\begin{array}{c}\text { Nừa cạnh } \\
\text { dưới sắp lại }\end{array}$} \\
\hline 3 & 7 \\
3 & 8 \\
3 & 4 \\
4 & 3 \\
4 & 8 \\
4 & 6 \\
6 & 7 \\
6 & 4 \\
6 & 8 \\
7 & 6 \\
7 & 8 \\
7 & 3 \\
8 & 3 \\
8 & 7 \\
8 & 6 \\
8 & 4 \\
\hline
\end{tabular}

Bảng 3

\begin{tabular}{|c|c|c|}
\hline \multicolumn{3}{|c|}{ Các mă̆t "Trên" } \\
\hline & ID & ID \\
Mặt & 1 & 2 \\
\cline { 2 - 3 } "Trên" & 1 & 2 \\
1 & 2 & 3 \\
& 3 & 4 \\
& 4 & 1 \\
\hline \multirow{2}{*}{ Mặt } & 1 & 6 \\
"Trên" & 6 & 7 \\
2 & 7 & 2 \\
& 2 & 1 \\
\hline & 1 & 4 \\
Mặt & 4 & 3 \\
"Trên" & 3 & 7 \\
3 & 7 & 6 \\
& 6 & 1 \\
\hline Mặt & 2 & 7 \\
"Trên" & 7 & 3 \\
4 & 3 & 2 \\
\hline
\end{tabular}

Các mặt "Dưới"
\begin{tabular}{|c|c|c|}
\hline & ID & ID \\
Mặt & 1 & 2 \\
\cline { 2 - 3 } "Dưới" & 3 & 7 \\
1 & 7 & 6 \\
& 6 & 4 \\
& 4 & 3 \\
\hline Mặt & 3 & 8 \\
"Dưới" & 8 & 7 \\
2 & 7 & 3 \\
\hline Mặt & 3 & 4 \\
"Dưới" & 4 & 8 \\
3 & 8 & 3 \\
\hline Mặt & 4 & 6 \\
"Dưới" & 6 & 8 \\
4 & 8 & 4 \\
\hline Mặt & 6 & 7 \\
"Dưới" & 7 & 8 \\
5 & 8 & 6 \\
\hline
\end{tabular}

\begin{tabular}{|c|c|c|}
\hline & ID & ID \\
Mặt & 1 & 2 \\
\cline { 2 - 3 } "Đứng" & 1 & 6 \\
1 & 6 & 4 \\
& 4 & 1 \\
\hline Mặt & 1 & 5 \\
"Đứng" & 5 & 6 \\
2 & 6 & 1 \\
\hline Mặt & 1 & 4 \\
"Đứng" & 4 & 5 \\
3 & 5 & 1 \\
\hline Mặt & 4 & 6 \\
"Đứng" & 6 & 5 \\
4 & 5 & 4 \\
\hline
\end{tabular}




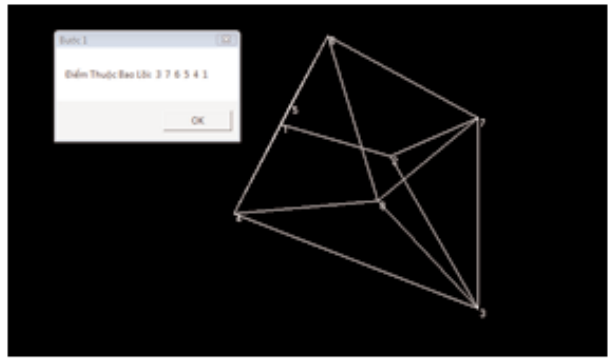

Bước 2: Chọn Zmax Và Zmin

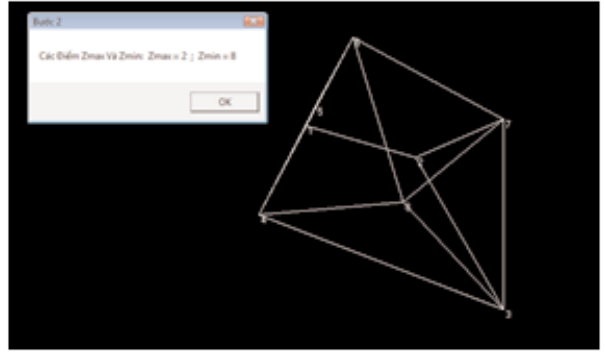

Bước 3: Tìm và sắp xếp theo danh sách nửa cạnh
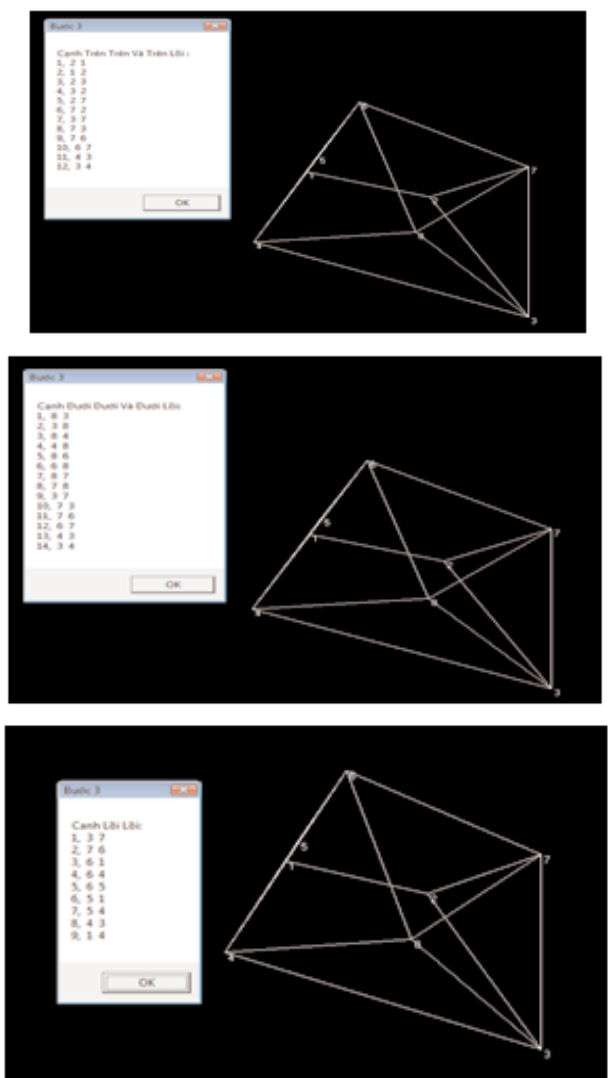

Bước 4: Tìm các cạnh "Đứng”

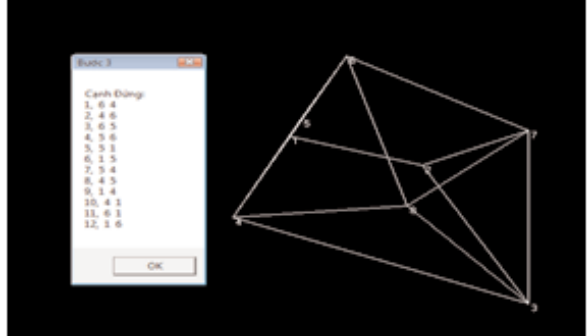

Bước 5: Kêt quả thu được các mặt

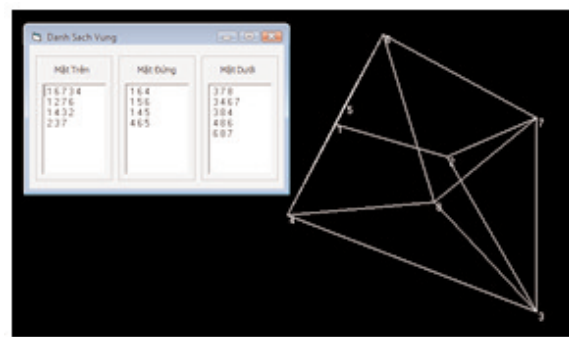

\section{Kết luận}

Bài toán tạo mặt cho các đối tượng trong không gian hết sức phức tạp. Nội dung bài báo này đã đề xuất một phương pháp có thể sử dụng khi nghiên cứu đối tượng $3 \mathrm{D}$. Hướng giải quyết bài toán tạo mặt này là phân loại các cạnh của khối 3D sau đó chiếu chúng về mặt phẳng. Việc phân loại này nhằm muc đích giải bài toán tạo mặt cho khối bằng cách tách bài toán không gian $3 D$ thành các bài toán trong không gian $2 D$ và sủ dụng bài toán tạo topo tìm vùng trong mặt phẳng để tạo mặt cho đối tương $3 D$.

Cùng với các nghiên cứu trong công trình [8], nội dung bài báo này góp một công cụ có thể sử dụng khi nghiên cứu giải bài toán $3 \mathrm{D}$ không gian được mô hình hóa từ dữ liệu là các điểm, đoạn (cạnh), mặt và khối. $\bigcirc$

\section{Tài liệu tham khảo}

[1]. Trần Thùy Dương (2005), "Phân tích biện pháp ánh xạ trong môi trường đồ họa", Tạp chí khoa học kỹ thuật Mỏ - Địa chất. 11, tr. 4749.

[2]. Trần Thùy Dương (2006), "Một giải pháp xử lý trường hợp biên trong bài toán tạo Topology", Tạp chí Khoa học kỹ thuật Mỏ - Địa chất. 14 , tr. 88-91. 
[3]. Trần Thùy Dương (2007), Nghiên cứu xây dựng công nghệ thành lập bản đồ số độ cao trong điều kiện Việt Nam, Luận án Tiến sĩ kỹ thuật, Trường Đại học Mỏ - Địa chất, Hà Nội.

[4]. Phạm Thế Huynh (2014), "Một cách tiếp cận mới trong việc giải quyết bài toán biên tập thửa đất sử dụng cấu trúc dữ liệu danh sách cạnh liên kết kép", Tạp chí khoa học Đo đạc và Bản đồ. 20, tr. 14-18.

[5]. Đinh Hải Nam (2009), "Nghiên cứu cấu trúc dữ liệu và thuật toán tạo Topology phục vụ cho công tác xây dựng cơ sở dữ liệu và quản lý

\section{Summary}

\section{A method of creating faces for 3D objects}

\section{Le Quang Hung, Resource Enviroment and Materials Technology Joinstock Company}

Tran Thuy Duong, Hanoi University of Mining and Geology

In the plane, the concept of the area used to describe a closed polygon is made up of the original data including the base elements, which are points and segments. To find and manage it, people use the problem of creating regions. The surface of a 3D object consists of a set of faces that are each modeled by a closed flat polygon. These surfaces are similar to areas in the plane but are represented in 3D space. The problem of creating areas in the plane has been studied and solved by the theory and practice applied to delineate and calculate the land area in a cadastral map. Based on this problem, the research paper proposes a surface creation method to describe the surface of $3 \mathrm{D}$ objects. This problem uses the principle of an edge with only two adjacent sides; from one edge, find the next edge. This problem will classify the edges of the 3D cube, then project them to the plane. The classification aims to simplify the spatial problem by converting it into a problem solving on the plane. However, when projecting the edges towards the plane, arranging the edges in the same order as in space is not a simple matter. This study shows that the above arrangement can be done when the faces meet certain conditions. The problem of creating a region on a plane creates surfaces corresponding to the surface of $3 \mathrm{D}$ objects in space. $\mathrm{O}$

\section{BÀN VỀ CHUYỂN ĐỘ CAO GEOID.......}

(Tiếp theo trang 6)

\section{Summary}

\section{About the conversion of a quasigeoid height into a geoid height}

\section{Ha Minh Hoa, Vietnam Institute of Geodesy and Cartography}

The research on conversion of the geoid height into the quasigeoid height is one of directions of research in physical geodesy. This article presentes theoretical research result by this direction and some research experiments results on the world. The research experiments results showed that difference between the geoid height and the quasigeoid height is quite big and at present not satisfied modern requirement of construction of geoid/quasigeoid model. That shows that the mass distribution between Earth physical surface and geoid still is difficult, at present still not exactly modeled. $\bigcirc$ 